\title{
Auditing the Accuracy of Medical Diagnostic Coding based on International Classification of Diseases, Tenth Revision
}

\author{
Seyed Hadi Mirhashemi ${ }^{1}$, Nahid Ramezanghorbani² ${ }^{2}$ Farkhondeh Asadi ${ }^{3,}{ }^{*}$ and Mehrnaz Hajiabedin Rangraz ${ }^{2}$ \\ 1 Department of General Surgery, Loghman Hakim Hospital, Shahid Beheshti University of Medical Sciences, Tehran, Iran \\ 2 Department of Development and Coordination of Scientific Information and Publications, Deputy of Research and Technology, Ministry of Health and Medical \\ Education, Tehran, Iran \\ ${ }^{3}$ Department of Health Information Technology and Management, School of Allied Medical Sciences, Shahid Beheshti University of Medical Sciences, Tehran, Iran
}

* Corresponding author: Farkhondeh Asadi, Department of Health Information Technology and Management, School of Allied Medical Sciences, Shahid Beheshti University of Medical Sciences, Tehran, Iran. Tel: +982122747373; Email: asadifar@sbmu.ac.ir

Received 2020 August 05; Revised 2020 August 28; Accepted 2020 September 19.

\begin{abstract}
Background: Medical diagnostic coding is used for the ease of retrieval and accuracy of medical information classification in health information systems. This information is the main source of decision making for health managers and policymakers in planning, epidemiological, and medical research at different levels.

Objectives: The present study aimed to audit the accuracy of the ICD-10 (International Classification of Diseases, Tenth Revision) medical diagnosis code.

Methods: The present cross-sectional study was performed on a sample of 692 hospitalized cases in 9 educational centers affiliated to Shahid Beheshti University of Medical Sciences in the first half of 2020. The content validity of the checklist was determined in this study, and the obtained data were analyzed in SPSS software using descriptive statistics.

Results: The average accuracy of coding for the main medical diagnoses across all subjects was $70 \%$, signifying that $30 \%$ of medical records contain coding errors. The highest and least accuracy values of diagnostic coding were $80 \%$ and $47 \%$, respectively. The application of standard abbreviations and file legibility were recognized as variables affecting code accuracy. The highest precision percentage of codes attributed to other medical diagnoses, including ICD-10-based comorbidity and complication, was in $84 \%-85 \%$ of the participants.

Conclusion: Given the importance of all-encompassing coding in retrieving medical information, research, and macro-health policymaking, the coding accuracy audit must be conducted on a regular basis. The interaction between coders and healthcare providers, coders' training, and improving the documentation process exerts a significant impact on the enhancement of coding accuracy.
\end{abstract}

Keywords: Coding accuracy, Coding audit, Coding quality, ICD-0, Medical diagnosis

\section{Background}

Medical records are valuable sources of information regarding the type and nature of the disease, outcomes, implemented measures, and treatment consequences. The importance of this resource lies in treatment programs, strategic planning in the field of medical care, as well as the evaluation and review of issues related to the management of diseases (1). Summarizing and placement of key case files in the health information system of health care centers is the key decisionmaking basis for health care managers and policymakers at different levels. In fact, information retrieval from the database gives policymakers remarkable insight into the incidence and prevalence of the disease and its development and enables them to take the necessary measures to reduce, track, or allocate the resources (2).

This would not be realized unless the information is properly organized and classified. This kind of information provides a golden opportunity for the implementation of data mining strategies in an attempt to improve outcomes and assess the varied impacts of these strategies on the growing healthcare environment. Despite the development of new analytical methods and symbolic improvement of computational power, the underlying basis for these analyses is the structured data coding for diseases and procedures as one of the key components (3).

The most significant purpose of coding is the facilitation of data retrieval and classification of morbidity and mortality. According to the World Health Organization (WHO), the purpose of grouping different diseases is not only the provision of accurate statistics of morbidity and mortality but also the presentation of useful information to establish diagnoses in clinical settings (4). Coding is a factor directly related to data quality. Medical coding specialists encrypt medical records to retrieve information concerning diseases and possible injuries (5).

These codes can provide reliable data for evaluating and improving the quality of patient care, helping to better understand complications, and designing robust clinical algorithms for disease and care outcomes (6). In new health care systems, referral to specific treatments depends on the proper allocation of diagnostic codes. Lack of correct codes will result in the loss of the identified therapeutic 
pathways for patients (7). Given the widespread and varied use of disease coding, therapeutic measures involved, and its importance, it is indispensable to ensure the accuracy of the performed coding.

Numerous studies have been conducted on the quality and accuracy of coding which yielded contradictory results. Some of these findings pointed to poor coding quality, while some others demonstrated good results (8-14). In their study, Thomas et al1. indicated the low accuracy of diagnostic codes for the identification of patients with cruciate ligament injury (15). Wang et al.1 also cited poor code levels as an indicator of treatment designation (11).

Xiao et al. ${ }^{1}$ performed a study entitled "Using the International Classification of Disease Codes to Identify Pancreatic Diseases". The results of the mentioned study pointed out that in 24 cohort studies involving 18,106 patients, sensitivity and specificity estimates for International Classification of Diseases (ICD) code for acute and chronic pancreatitis were 0.85 and 0.96 , respectively. The correct prediction value of ICD codes for acute and chronic pancreatitis was 0.71 which increased to 0.78 when only applied for acute pancreatitis. The final results of the study showed that approximately 3 out of 10 patients with acute and chronic pancreatitis are misdiagnosed using ICD codes (16).

Along the same lines, Liu' ${ }^{1}$ (2018) carried out a study entitled "A Review of the Accuracy of Disease Codes in Patients with Sarcoma". The results of the referred study indicated that out of 1,237 patient codes reviewed, $61.8 \%$ were correctly coded, $16.8 \%$ received non-cancer codes, and $21.4 \%$ received incomplete codes. The stated study also emphasized that the ambiguous nature of disease definitions can extend coding errors (17).

In their study entitled "Accuracy of Diagnostic Codes to Identify Patients with Syncope in the Emergency Department", Forlene et al. ${ }^{1}$ found that the accuracy of the diagnostic code for identifying this group of patients was $0.63 \%$ with a $95 \%$ confidence interval (18). In the same context, the findings of a study conducted by Ang Procerte ${ }^{1}$ entitled "Verifying the accuracy of diagnostic codes for sarcoidosis patients" revealed that out of 224 cases of sarcoidosis, only $61.22 \%$ demonstrated positive code values (19).

Based on the aforementioned results, coding error mostly leads to incorrect classification of diseases, and planning based on incorrect information would result in inappropriate outcomes. Trust in data with unclear or poor classification quality places both health care providers and managers at risk in planning, epidemiological research, and medical research. So far, no comprehensive study has been performed on the accuracy of coding for ICD-10 based medical diagnoses in hospitals affiliated to Shahid Beheshti University of Medical Sciences.

\section{Objectives}

The present study aimed to audit the accuracy of Medical Diagnoses Coding based on ICD-10 performed in the educational hospitals affiliated to Shahid Beheshti University of Medical Sciences.

\section{Methods}

This cross-sectional study aimed to audit the accuracy of Medical Diagnoses Coding based on ICD-10 in nine educational hospitals affiliated to Shahid Beheshti University of Medical Sciences. The study population consisted of all records of patients admitted to research units in the first half of 2019. The current research was conducted in the following stages:

\subsection{Compiling a checklist to audit the accuracy of medical diagnostic coding \\ A search was carried out on Ovid, Scopus,} PubMed, Science Direct, and Google Scholar databases using the following keywords: "ICD Code, Coding Audit, Coding Quality, Coding Accuracy, International Classification of Diseases, Quality of diagnosis".Based on this literature search and the revision of ICD-10 coding requirements and rules, the researcher-made checklist was developed. The first part of the checklist (6 questions)investigated the factors affecting coding accuracy, and the second part (13 questions) examined the coding accuracy of medical diagnoses (main condition, other conditions). The content validity of the checklist was confirmed using the opinions of 10 experts in health information management.

\subsection{Sample size determination}

To achieve this objective, a pilot study was performed based on the checklists designed at each center to check the accuracy of the diagnostic codes and compare the discrepancy ratios. Thereafter, at a confidence interval of $95 \%$, the sample size was estimated at 692 cases in 9 educational centers using the following formula:

$$
n=\frac{Z^{2}\left(\frac{\alpha}{2}\right) P(1-P)}{\alpha 2}=692
$$

In this formula, $\mathrm{n}$ is the sample size under the study, $\mathrm{Z}$ is normal variance $(1.96), \mathrm{P}$ is contrast ratio, and $\alpha$ is coefficient $(0.05)$.

\subsection{Collecting the required data}

At this stage, questions about the factors affecting coding accuracy were completed through interviews with coders, observation, and careful review of the environment and medical records. To verify the coding, the required files were first abstracted, and the dedicated codes were separately recorded. The abstracts were then coded again. The results were 
compared with baseline codes, and contrast ratios were specified. The obtained data were analyzed in SPSS software using descriptive statistics.

\section{Results}

Based on the results of the present study, the highest and lowest accuracy scores of Main Conditions coding were obtained at $80 \%$ and $47 \%$, respectively. The average coding accuracy of all research units manifested $70 \%$ accuracy of the Main Conditions coding. This signifies that $30 \%$ of medical records are not coded correctly for several reasons (Figure 1). The highest accuracy of codes assigned to other Conditions, including comorbidity and complication based on ICD-10, was reported to be within the range of $5-84 \%$.

Checking the accuracy of coding in all research units indicates that $57 \%$ of other Conditions are coded correctly, that is to say, other Conditions are not coded correctly in $43 \%$ of medical records (Figure 2).

The factors affecting the coding accuracy, which are presented in Table 1, was another significant finding of the current study. As illustrated in Table 1, coding errors arise from non-adherence to the following factors: medical records illegibility(30\%), the use of non-standard abbreviations (10\%), and nonconformity of diagnostic principles with physicians (33\%).

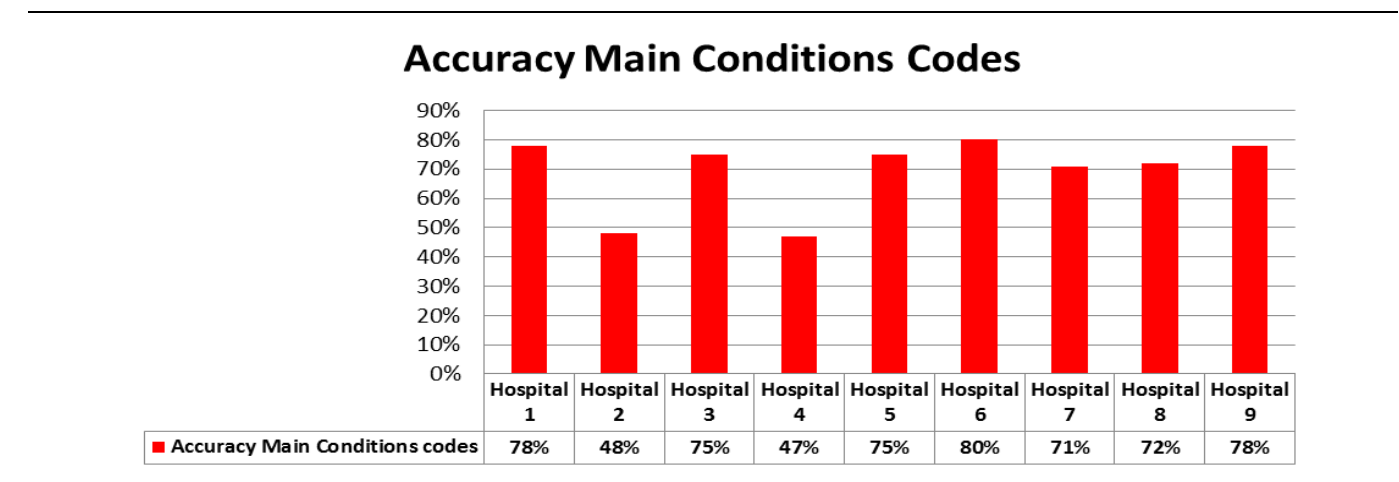

Figure 1. Percentage distribution of Accuracy of Codes assigned to Main Conditions based on ICD-10 in research units

\section{Accuracy Other Conditions Codes}

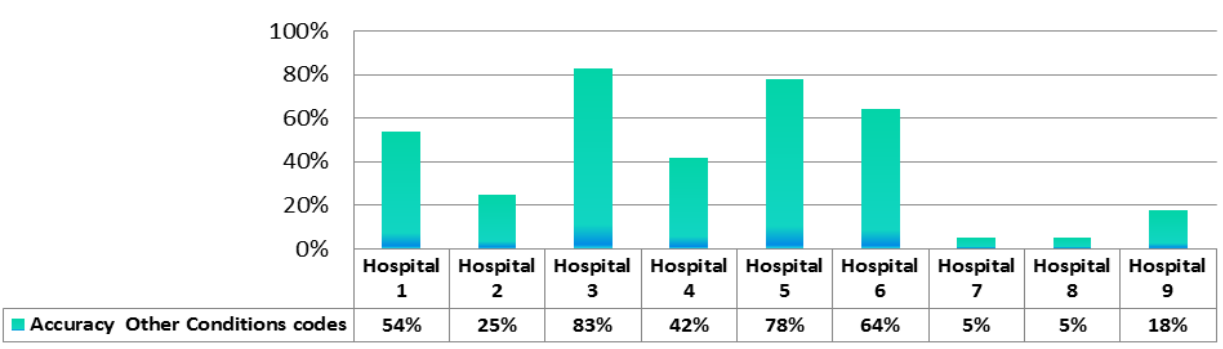

Figure 2. Percentage distribution of codes assigned to other conditions, including comorbidity and complication based on ICD-10 in research units

\begin{tabular}{lccc}
\hline Table 1. Percentage Distribution of Factors Related to the Accuracy of Medical Conditions coding based on ICD-10 in Research Units \\
\hline Relevant Factors & $\begin{array}{c}\text { Using both of ICD-10 lists } \\
\text { for code assigning }\end{array}$ & $\begin{array}{c}\text { Observing the } \\
\text { diagnostic principles }\end{array}$ & $\begin{array}{c}\text { Clear and standard } \\
\text { abbreviations }\end{array}$ \\
\hline Research Units & $\mathbf{N ( \% )}$ & $\mathbf{N ( \% )}$ & $\mathbf{N ( \% )}$ \\
\hline $\mathbf{1}$ & $100(100)$ & $70(70)$ & $92(92)$ \\
$\mathbf{2}$ & $72(100)$ & $54(75)$ & $66(93)$ \\
$\mathbf{3}$ & $70(100)$ & $47(67)$ & $61(91)$ \\
$\mathbf{4}$ & $45(10)$ & $29(65)$ & $41(91)$ \\
$\mathbf{5}$ & $95(10)$ & $64(67)$ & $87(92)$ \\
$\mathbf{6}$ & $76(85)$ & $65(72)$ & $66(92)$ \\
$\mathbf{7}$ & $70(100)$ & $42(60)$ & $60(90)$ \\
$\mathbf{8}$ & $49(100)$ & $30(61)$ & $45(92)$ \\
$\mathbf{9}$ & $50(100)$ & $30(60)$ & $44(88)$ \\
Total & $627(705)$ & $431(597)$ & $56(70)$ \\
\hline
\end{tabular}




\section{Discussion}

As evidenced by the obtained results, the accuracy of Main Conditions codes was $\% 70$ with the error of $30 \%$. The accuracy of complication and co-morbidity diagnostic codes was reported as $57 \%$ and the error of this category of codes was obtained at \%43. Moreover, it was found that most of the factors related to the accuracy of the coding of medical diagnoses based on ICD-10 in research units were related to the assessment and application of ICD-10 instructions for code allocation.

The accuracy and errors of the Main Conditions codes are almost in agreement with the findings of a study conducted on coding accuracy in Kashan Educational Hospitals. In the present study, $77.3 \%$ of diagnostic codes were valid. 84 errors $(22.7 \%)$ were observed among the diagnostic codes, out of which $28(33.3 \%)$ cases were major errors and $56(66.7 \%)$ cases were minor ones. The application of coding instructions and nonuse of abbreviations significantly reduced the coding error. (20) The accuracy of the main diagnostic codes in Shahid Beheshti Educational Hospitals (70\%) has proved to be less, as compared to coding accuracy in Shiraz Educational Hospitals (81.3\%) (21), Tehran (82.2\%), Shahid Beheshti (85\%), and Iran (82.2\%) in 2006. (22)

In the same direction, the results of a study on the accuracy of obesity codes at Lausanne Hospital in Switzerland also showed that ICD-10 obesity codes have very low sensitivity (7.75\%), high specificity (99\%), and moderate positive value (66\%). In general, the accuracy of the diagnostic codes reported in the field of obesity is higher (23). In this regard, numerous studies have been conducted on some diseases in other countries.

As for the inaccurate selection of the main condition by the coders and their human error, Lucyk et al. (2017) pointed out that the diversity in the interpretation of data is a major obstacle to the quality of data in documentation from admission to discharge. The data interpreted and coded by the coders must only come from sources which are explicitly documented by physicians. The use of nonstandard abbreviations poses a threat to quality. The legibility of documents also affects the quality of codes. In most cases, coders consult with their colleagues to interpret the handwriting, make a decision based on their experience with each physician's writing style, or adopt the "best guess" method to code the documents, which may cause errors in the selection of diagnosis. In other words, insufficient data and non-specialized or inaccurate documents complicate the coding process, give rise to coders' uncertainty, and leads to inaccurate identification and coding of diagnosis (24).

Numerous studies have been conducted on the accuracy of coding in other countries. The evaluation of the accuracy of diagnostic coding in the studies conducted by Xiao et al. on chronic acute pancreatitis (10), Liu in patients with sarcoma (17), and Worfellan et al. on patients with syncope (18) is not significantly different from the present study.

In a cohort study performed by Hamilton et al., out of 25 diagnostic codes assigned to medical records, only 18 cases were accurate, that is to say, the accuracy of diagnostic code was almost $72 \%$ (25). These findings confirm the results of the present study. Accurate registration and coding seem crucial since other conditions, including comorbidity and complication, are part of the final diagnosis. Some studies showed that other conditions are not recorded in a specific location in the medical record, and others reported non-registration as 63\% (26).

In the present study, the accuracy of other conditions codes was reported as $57 \%$. This makes it impossible to accurately measure patients or associated disorders when assessing the burden of disease over society. Diagnostic codes can be the primary source of hospital data for hospital budgeting and quality improvement. Therefore, the inaccuracy of the diagnostic codes increases when the coders limit their scope to the use of discharge summaries. Moreover, if an unfamiliar medical practitioner uses the same summary, it may lead to poor accuracy of diagnosis.

Tsopra et al. suggested that if the data needed to summarize patient discharge by specialist physicians were based on patient records, reports, and patient notes, it could reduce hospital costs by 1.8-16.5 million pounds (27); nonetheless, the results of different studies may not actually be comparable. Overall, it seems that the accuracy of the diagnostic codes or the errors in diagnostic coding in nine educational hospitals affiliated to Shahid Beheshti University of Medical Sciences is not significantly different from other studies.

Medical records affect the coded data for a range of reasons, including unclear documentation and variations in the description of diagnoses. Therefore, the use of these standards can be effective in diagnostic coding and audits to improve the quality of coded data (28). The present study also confirmed the impact of non-compliance with diagnostic principles (31.6\%) and medical records legibility $(17.2 \%)$ on the given codes. It is important to identify clinical and management points during treatment, review the ICD-10-based diagnostic code, use multiple diagnostic codes, and train staff in this area to improve the accuracy of the clinical codes.

The identified problems reflect the challenges posed to the validity of clinical codes when dealing with other complex (1) and common diseases (29). Multiple studies also confirmed the counseling and implementation of training programs for service providers and coders in the field of clinical practice documentation and coding in order to visualize the accurate clinical images and reduce coding errors in 
medical records $(30,31)$.

In their study entitled "Quality of Medical Information Determines Quality of Diagnostic Code", Maryati et al. stated that high quality of medical information leads to better diagnostic coding (about $73.8 \%$ ), and poor quality of medical information results in poor diagnostic coding (about 36\%) (32). Therefore, it is essential that researchers assess this potential error when evaluating other diseases. Lucyk also argued that information that is interpreted and coded by a coder should only come from sources which are explicitly documented by physicians. Document legibility affects code quality.

All things considered, coding is a complex process which becomes even more complicated due to such issues as inadequate and incomplete data and inaccurate documentation, which in turn, gives rise to uncertainty in coding and may lead to misidentification of codes and wrong coding (33). Santos believes that factors that may affect coding quality include appropriate interaction between coders and healthcare providers, coder training, coding and documentation process by physicians, coding environment and workload, as well as access to reference and required books needed for code allocation (34).

Kiongo (2018) assessed the role of training on the quality of coding in Kenya and concluded that following the training, coding accuracy was increased from $55 \%$ to $77 \%$, coding completeness was enhanced from $96.8 \%$ to $98.9 \%$, and the quality of coding the external causes of injuries was promoted from $67.6 \%$ to $86.5 \%$. According to Kionogo, the disease classification system used in the ICD is a complex model that requires continuous training (35).

Therefore, the role of effective and continuous training in coders should not be overlooked since their unawareness of the basic coding rules results in incorrect coding. The comparison of the present research with other studies makes clear that diagnostic coding can be enhanced in a myriad of ways, many of which are similar. It is worth mentioning that one of the notable limitations of the current study was the mere assessment of educational hospitals affiliated to Shahid Beheshti University of Medical Sciences. It is suggested that similar studies be performed across all provinces in the country.

\section{Conclusion}

Given the importance of all-encompassing coding in retrieving medical information, research, and macro-health policymaking, the coding accuracy audit must be conducted on a regular basis. The interaction between coders and healthcare providers, coders' training, and improving the documentation process exerts a significant impact on the enhancement of coding accuracy.

\section{Acknowledgements}

The present article was extracted from a research project that was supported by Shahid Beheshti University of Medical Science. The authors' deepest appreciation goes to the Vice- Chancellor's Office in Treatment Affairs and the Vice- Chancellor's Office in Research Affairs for their valuable support since the commencement of the current study.

\section{Footnotes}

Authors' Contribution: All authors contributed to all aspects of the article.

Ethical Approval: The present article was extracted from a research project approved by the Ethics Committee of SBMU (IR.SBMU.RETECH.REC.1397. 1014)

Funding/Support: The present study was financially supported by Shahid Beheshti University of Medical Sciences [Research project code: 15152].

\section{References}

1. Thomas J. Medical records and issues in negligence. Indian J Urol. 2009;25(3):384-8. doi: 10.4103/0970-1591.56208. [PubMed: 19881136].

2. Yazdi-Feyzabadi V, Emami M, Mehrolhassani MH. Health information system in primary health care: the challenges and barriers from local providers' perspective of an area in Iran. Int J Prev Med. 2015;6:57. doi: 10.4103/2008-7802.160056. [PubMed: 26236444].

3. Weiner MG. POINT: Is ICD-10 diagnosis coding important in the era of big data? Yes. Chest. 2018;153(5):1093-5. doi: 10.1016/j.chest.2018.01.025. [PubMed: 29410203].

4. Clark LA, Cuthbert B, Lewis-Fernández R, Narrow WE, Reed GM. Three approaches to understanding and classifying mental disorder: ICD-11, DSM-5, and the national institute of mental health's research domain criteria (RDoC). Psychol Sci Public Interest. 2017;18(2):72-145. doi: 10.1177/1529100617727266. [PubMed: 29211974].

5. Serinoglu L, Yarar O, Eti CM, Puturgeli T, Vayisoglu Y, Farsak M, et al. The importance of ICD-10 applications on daily practice. Medicine. 2018;7(4):873-7. doi: 10.5455/medscience.2018. 07.8843 .

6. Bowman SE. Why ICD-10 Is worth the trouble. J AHIMA. 2008;79(3):24-9. [PubMed: 18421998].

7. Treede RD, Rief W, Barke A, Aziz Q, Bennett MI, Benoliel R, et al. Chronic pain as a symptom or a disease: the IASP classification of chronic pain for the international classification of diseases (ICD-11). Pain. 2019;160(1):19-27. doi: 10.1097/j. pain.0000000000001384. [PubMed: 30586067].

8. Storesund A, Haugen AS, Hjortas M, Nortvedt MW, Flaatten H, Eide GE, et al. Accuracy of surgical complication rate estimation using ICD-10 codes. Br J Surg. 2019;106(3):236-44. doi: 10.1002/bjs.10985. [PubMed: 30229870].

9. Bosco-Levy P, Duret S, Picard F, Dos Santos P, Puymirat E, Gilleron V, et al. Diagnostic accuracy of the international classification of diseases, tenth revision, codes of heart failure in an administrative database. Pharmacoepidemiol Drug Saf. 2019;28(2):194-200. doi: 10.1002/pds.4690. [PubMed: 30395375].

10. Xiao AY, Tan ML, Plana MN, Yadav D, Zamora J, Petrov MS. The use of international classification of diseases codes to identify patients with pancreatitis: a systematic review and metaanalysis of diagnostic accuracy studies. Clin Transl Gastroenterol. 2018;9(10):191. doi: 10.1038/s41424-0180060-1. [PubMed: 30287807].

11. Wong J, Abrahamowicz M, Buckeridge DL, Tamblyn R. 
Assessing the accuracy of using diagnostic codes from administrative data to infer antidepressant treatment indications: a validation study. Pharmacoepidemiol Drug Saf. 2018;27(10):1101-11. doi: 10.1002/pds.4436. [PubMed: 29687504].

12. Li L, Binney LE, Luengo-Fernandez R, Silver LE, Rothwell PM. Temporal trends in the accuracy of hospital diagnostic coding for identifying acute stroke: A population-based study. Eur Stroke J. 2020;5(1):26-35. doi: 10.1177/2396987319881017. [PubMed: 32232167].

13. Muzerengi S, Rick C, Begaj I, Ives N, Evison F, Woolley RL, et al. Coding accuracy for Parkinson's disease hospital admissions: implications for healthcare planning in the UK. Public Health. 2017;146:4-9. doi: 10.1016/j.puhe.2016.12.024. [PubMed: 28404473].

14. Delate T, Jones AE, Clark NP, Witt DM. Assessment of the coding accuracy of warfarin-related bleeding events. Thromb Res. 2017;159:86-90. doi: 10.1016/j.thromres.2017.10.004. [PubMed: 29035718]

15. Sanders TL, Pareek A, Desai VS, Hewett TE, Levy BA, Stuart MJ, et al. Low accuracy of diagnostic codes to identify anterior cruciate ligament tear in orthopaedic database research. Am J Sports Med. 2018;46(12):2894-8. doi: 10.1177/036354651 8790507. [PubMed: 30125126].

16. Xiao AY, Tan ML, Plana MN, Yadav D, Zamora J, Petrov MS. Correction: the use of international classification of diseases codes to identify patients with pancreatitis: a systematic review and meta-analysis of diagnostic accuracy studies. Clin Transl Gastroenterol. 2018;9(11):209. doi: 10.1038/s41424018-0078-4. [PubMed: 30464255].

17. Lyu HG, Stein LA, Saadat LV, Phicil SN, Haider A, Raut CP, et al. Assessment of the accuracy of disease coding among patients diagnosed with sarcoma. JAMA Oncol. 2018;4(9):1293-5. doi: 10.1001/jamaoncol.2018.2979. [PubMed: 30098150].

18. Furlan L, Solbiati M, Pacetti V, Dipaola F, Meda M, Bonzi M, et al. Diagnostic accuracy of ICD-9 code 780.2 for the identification of patients with syncope in the emergency department. Clin Auton Res. 2018;28(6):577-82. doi: 10.1007/s10286-018-0509-z. [PubMed: 29435866].

19. Ungprasert P, Matteson EL, Crowson CS. Accuracy of diagnostic coding for sarcoidosis in electronic databases: a populationbased study. Lung. 2017;195(6):713-5. doi: 10.1007/s00408017-0054-x. [PubMed: 28993879].

20. Farzandipour M, Sheikhtaheri A. Accuracy of diagnostic coding based on ICD-10. Feyz. 2009;12(4):68-77.

21. Mahmudzade Sagheb Z. Determination of coding accuracy in the training hospitals of Shiraz University of Medical Sciences. [Master Thesis]. Tehran: School of Management and Medical Information Sciences of Iran University of Medical Sciences; 1995.

22. Ahmadi M, Alipour J. Adherance to quality elements for coding of diagnoses and procedures. Hakim Res J. 2009;12(1):11-8.

23. Khalatbari-Soltani S, Waeber G, Marques-Vidal P. Diagnostic accuracy of undernutrition codes in hospital administrative discharge database: improvements needed. Nutrition. 2018;55-56:111-5. doi: 10.1016/j.nut.2018.03.051. [PubMed: 29980090].

24. Lucyk K, Tang K, Quan H. Barriers to data quality resulting from the process of coding health information to administrative data: a qualitative study. BMC Health Serv Res. 2017;17(1):766. doi: 10.1186/s12913-017-2697-y. [PubMed: 29166905].

25. Hamilton F, Arnold D. Accuracy of clinical coding of pleural empyema: a validation study. J Eval Clin Pract. 2020;26(1):7980. doi: 10.1111/jep.13184. [PubMed: 31115122].

26. Powell H, Lim LY, Heller RF. Accuracy of administrative data to assess comorbidity in patients with heart disease: an Australian perspective. J Clin Epidemiol. 2001;54(7):687-93. doi: 10.1016/s0895-4356(00)00364-4. [PubMed: 11438409].

27. Tsopra R, Peckham D, Beirne P, Rodger K, Callister M, White H, et al. The impact of three discharge coding methods on the accuracy of diagnostic coding and hospital reimbursement for inpatient medical care. Int J Med Inform. 2018;115:35-42. doi: 10.1016/j.ijmedinf.2018.03.015. [PubMed: 29779718].

28. Alonso V, Santos JV, Pinto M, Ferreira J, Lema I, Lopes F, et al. Health records as the basis of clinical coding: Is the quality adequate? A qualitative study of medical coders' perceptions. Health Inf Manag. 2020;49(1):28-37. doi: 10.1177/18333 58319826351. [PubMed: 30744403].

29. Taylor K, Henderson S. 112 Challenges of clinical coding: an audit of the accuracy of primary diagnosis coding in a specialist palliative care unit. BMJ Support Palliat Care. 2019;9(Suppl 1):A49-50. doi: 10.1136/bmjspcare-2019ASP.135.

30. Castaldi M, McNelis J. Introducing a clinical documentation specialist to improve coding and collectability on a surgical service. J Healthc Qual. 2019;41(3):e21-9. doi: 10.1097/ JHQ.0000000000000146. [PubMed: 31094954].

31. Shaheen NA, Manezhi B, Thomas A, AlKelya M. Reducing defects in the datasets of clinical research studies: conformance with data quality metrics. BMC Med Res Methodol. 2019;19(1):98. doi: 10.1186/s12874-019-0735-7. [PubMed: 31077148]

32. Maryati W, Rahayuningrum IO, Ismayani A. Quality of medical information determine the quality of diagnosis code. Int $J$ Public Health Sci. 2019;8(3):326-31. doi: 10.11591/ijphs. v8i3.20236.

33. Tang KL, Lucyk K, Quan H. Coder perspectives on physicianrelated barriers to producing high-quality administrative data: a qualitative study. CMAJ Open. 2017;5(3):E617-22. doi: 10.9778/cmajo.20170036. [PubMed: 28827414].

34. Santos S, Murphy G, Baxter K, Robinson KM. Organisational factors affecting the quality of hospital clinical coding. Health Inf Manag. 2008;37(1):25-37. doi: 10.1177/1833358308037 00103. [PubMed: 18245862].

35. Kiongo JG. Effects of training on quality of clinical coding at mbagathi county referral. Int J Innovat Educ Res. 2018; 6(1):231-7. doi: 10.31686/ijier.vol6.iss1.943. 